\title{
Phase-space distributions and the classical component of quantum observables
}

\author{
Alfredo Luis* \\ Departamento de Óptica, Facultad de Ciencias Físicas, Universidad Complutense, 28040 Madrid, Spain
}

(Received 9 March 2003; published 20 June 2003)

\begin{abstract}
We analyze the relation between the classical part of quantum observables and the distributions representing quantum states and observables on the classical phase space. We determine in which conditions such a relation can be established, and the proper phase-space distribution required for this purpose.
\end{abstract}

DOI: 10.1103/PhysRevA.67.064101

PACS number(s): 03.65.Sq, 03.65.Ca, 03.65.Ta

The representation of quantum states and observables by distributions on the classical phase space provides a distinguished tool to analyze fundamental aspects of the quantum physics, specially concerning the fuzzy boundary between the classical and quantum theories. For example, they can be used to determine how nonclassical is a quantum state (the so-called nonclassical depth) [1].

Some recent works have put forward a decomposition of quantum observables into the sum of classical and nonclassical components [2,3]. This splitting presents surprising and interesting properties concerning quantum fluctuations, uncertainty relations, and other fundamental aspects of the quantum theory [2-4]. In particular, it has been shown that the classical part of the linear momentum (with respect to position) coincides with a local average of the Wigner function.

This connection suggests a natural and fruitful relation between classical parts and classical-like description of quantum physics. In this work we study this correspondence in a more general framework by examining the case of arbitrary observables beyond linear momentum. On the one hand, when we consider arbitrary functions of momentum we find that the classical part is the local average of a phasespace distribution different from the Wigner function. (Naturally, for linear momentum such a distribution and the Wigner function give the same result.) On the other hand, if we consider arbitrary joint functions of position and momentum, we find that no phase-space correspondence of this kind can be established.

The decomposition of quantum observables $B$ into the sum of classical $B_{c}^{A}$ and a nonclassical $B_{q}^{A}$ components, $B$ $=B_{q}^{A}+B_{c}^{A}$, has been introduced in Refs. [2,3]. The classical component $B_{c}^{A}$ of the observable $B$ with respect to operator $A$ is defined as the operator commuting with $A$ that minimizes the mean deviation

$$
\mathcal{E}_{P}=\left\langle\left(B-B_{c}^{A}\right)^{2}\right\rangle
$$

where

$$
B_{c}^{A}=\sum_{a} B_{c}^{A}(a)|a\rangle\langle a|
$$

\footnotetext{
*Electronic address: alluis@ fis.ucm.es
}

and $|a\rangle$ are the eigenstates of $A$. The minimization of $\mathcal{E}_{P}$ leads to

$$
B_{c}^{A}(a)=\frac{\langle a|(B \rho+\rho B)| a\rangle}{2\langle a|\rho| a\rangle}=\frac{\langle(|a\rangle\langle a|B+B| a\rangle\langle a|)\rangle}{2\langle\mid a\rangle\langle a \mid\rangle},
$$

where $\rho$ is the density matrix of the system.

For definiteness, in what follows we focus on the case that the reference observable $A$ is the Cartesian position of a onedimensional system, $A=X$, while $B$ will be arbitrary in principle. Incidentally, when $A=X$, the classical parts are closely related to the de Broglie-Bohm approach to quantum mechanics, as discussed in Refs. [4,5]. Moreover, when $B$ is the linear momentum $P$, the classical part becomes proportional to the probability current density, which is a very intuitive result. In particular, for pure states $|\psi\rangle$

$$
P_{c}^{X}(x)=\hbar \frac{d}{d x} \arg \psi(x),
$$

where $\psi(x)=\langle x \mid \psi\rangle$ as usual.

In Refs. [2,3] it has been shown that $P_{c}^{X}$ can be suitably related to the Wigner function as a kind of a local expectation value of the form

$$
P_{c}^{x}(x)=\frac{\int d p p W(x, p)}{\int d p W(x, p)},
$$

where $W(x, p)$ stands for the Wigner function,

$$
W(x, p)=\frac{1}{2 \pi \hbar} \int d \xi e^{i p \xi / \hbar}\langle x-\xi / 2|\rho| x+\xi / 2\rangle,
$$

and $|x \pm \xi / 2\rangle$ are the eigenstates of $X$.

At this stage we recall that the Wigner function is not the unique description of quantum physics on phase space, and many other choices are possible [6-12]. In our context, it can be easily seen that the same relation (5) is satisfied by very different phase-space distributions. For example, this is the case of the family of functions introduced in Ref. [7] 


$$
W_{f}(x, p)=\frac{1}{(2 \pi \hbar)^{2}} \iiint d \theta d \tau d u f(\theta, \tau) e^{-i x \theta / \hbar} e^{i p \tau / \hbar} e^{i \theta u / \hbar}\langle u-\tau / 2|\rho| u+\tau / 2\rangle,
$$

provided that

$$
\left.\frac{\partial f}{\partial \tau}\right|_{\tau=0}=0
$$

As a further example, relation (5) is also satisfied by the de Broglie-Bohm distribution for pure states $[5,8]$

$$
W_{\mathrm{BB}}(x, p)=|\psi(x)|^{2} \delta\left(p-\hbar \frac{d}{d x} \arg \psi(x)\right),
$$

where $\delta$ is the Dirac delta function.

After this ambiguity, we can ask whether the nonuniqueness persists when we consider other observables different from momentum or, on the contrary, the general case singles out a unique solution. To this end, let us consider the relation

$$
B_{c}^{X}(x)=\frac{\langle x|(B \rho+\rho B)| x\rangle}{2\langle x|\rho| x\rangle}=\frac{\int d p \mathcal{B}(x, p) \mathcal{W}(x, p)}{\int d p \mathcal{W}(x, p)},
$$

for arbitrary $B$ as a definition of the phase-space distribution $\mathcal{W}(x, p)$. The objective is to solve this relation for $\mathcal{W}(x, p)$. To this end, we will assume the natural equality

$$
\langle x|\rho| x\rangle=\int d p \mathcal{W}(x, p)
$$

so that Eq. (10) becomes

$$
\frac{1}{2}\langle x|(B \rho+\rho B)| x\rangle=\int d p \mathcal{B}(x, p) \mathcal{W}(x, p) .
$$

Incidentally, Eq. (12) implies a natural expression for the mean value of $B$

$$
\langle B\rangle=\int d p d x \mathcal{B}(x, p) \mathcal{W}(x, p) .
$$

Before proceeding further, let us note that relation (10) may be ambiguous since we have no prior assignment for the function $\mathcal{B}(x, p)$. Fortunately, this is not a difficulty, since in order to solve Eq. (10), it is sufficient to consider just operator functions $B(P)$ depending only on $P$. In such a case we can naturally assume that $\mathcal{B}(p)=B(p)$. Specifically, we consider the family of operators $B=e^{i y P / \hbar}$. Since $e^{i y P / \hbar}|x\rangle=\mid x$ $-y\rangle$, we get

$$
\langle x+y|\rho| x\rangle+\langle x|\rho| x-y\rangle=2 \int d p e^{i y p / \hbar} \mathcal{W}(x, p),
$$

so that by Fourier inversion we get

$$
\begin{aligned}
\mathcal{W}(x, p) & =\frac{1}{2 \pi \hbar} \operatorname{Re}\left(\int d y e^{-i y p / \hbar}\langle x|\rho| x-y\rangle\right) \\
& =\frac{1}{\sqrt{2 \pi \hbar}} \operatorname{Re}\left(e^{-i x p / \hbar}\langle x|\rho| p\rangle\right),
\end{aligned}
$$

where $|p\rangle$ are the eigenstates of $P$.

The first conclusion is that the solution is unique. The second conclusion is that the solution is not the Wigner function. The distribution (15) was introduced in Ref. [9], and it corresponds to $f=\cos [\theta \tau /(2 \hbar)]$ in Eq. (7) (while the Wigner function corresponds to $f=1$ ) [7]. Formally, $\mathcal{W}(x, p)$ is quite similar to the distribution introduced in Ref. [10] providing the instantaneous power spectrum of a temporal signal.

In Refs. [7,11,12] it is shown that the phase-space formalism defined by Eq. (15) provides the following correspondence between operators and functions required to fulfill the relation $(13)$ :

$$
\mathcal{B}(x, p)=x^{n} p^{m} \leftrightarrow B=\frac{1}{2}\left(P^{m} X^{n}+X^{n} P^{m}\right) .
$$

Once we have determined explicitly the function $\mathcal{W}(x, p)$ we can go backwards to discuss the assumptions adopted above. In the first place, it can be easily checked that the condition (11) is actually satisfied. Furthermore, we have also

$$
\langle p|\rho| p\rangle=\int d x \mathcal{W}(x, p)
$$

On the other hand, for the most general case when $B(X, P)$ depends both on $X$ and $P$, we can demonstrate that Eq. (12) is not compatible with Eq. (15). To show this, let us consider the case $\mathcal{B}(x, p)=j(x) g(p)$ where $j$ and $g$ are arbitrary functions. From Eq. (15), the right-hand side of Eq. (12) can be expressed as

$$
\frac{1}{2}\langle x|(\rho G J+J G \rho)| x\rangle,
$$

where the operators $J, G$ are defined as $J=j(X)$ and $G$ $=g(P)$, respectively. On the other hand, from the functionoperator correspondence (16) the operator $B$ is $B=(G J$ $+J G) / 2$, so that the left-hand side of Eq. (12) is

$$
\frac{1}{4}\langle x|[\rho(G J+J G)+(G J+J G) \rho]| x\rangle .
$$

Equations (18) and (19) are equal if and only if

$$
\langle x|\rho[J, G]| x\rangle=\langle x|[J, G] \rho| x\rangle,
$$

or, equivalently,

$$
\operatorname{tr}(\rho[|x\rangle\langle x|,[J, G]])=0 .
$$


For arbitrary $\rho$ this implies that the commutator $[J, G]$ commutes with every $|x\rangle\langle x|$ and, therefore,

$$
[J, G]=H(X),
$$

where $H(X)$ is an arbitrary function of $X$. This allows us to present the case $j(x)=x, g(p)=p^{2}$, with $[J, G]=2 i \hbar P$, as a suitable example for which Eq. (22) and, therefore, Eq. (12) are not satisfied. This does not exclude that there are some other particular joint functions of $x, p$ for which Eqs.
(22) and (12) are satisfied. This is the case of $j(x)$ arbitrary and $g(p)=p$, for which $H=-i \hbar d J / d x$ in Eq. (22), that includes the relevant example of the interaction Hamiltonian of a particle coupled to the electromagnetic field.

The conclusion is that, when we take as reference the observables $X$ or $P$, the classical part of the observable $B$ is a local average of the Margenau-Hill phase-space distribution provided that $[B, X]=0$ or $[B, P]=0$. Otherwise, for arbitrary joint functions of $X$ and $P$, the phase-space connection does not hold in general.
[1] C.T. Lee, Phys. Rev. A 44, R2775 (1991); N. Lütkenhaus and S.M. Barnett, ibid. 51, 3340 (1995); C.T. Lee, ibid. 52, 3374 (1995); A.F. de Lima and B. Baseia, ibid. 54, 4589 (1996); J. Janszky, M.G. Kim, and M.S. Kim, ibid. 53, 502 (1996).

[2] M.J.W. Hall, Phys. Rev. A 64, 052103 (2001).

[3] M.J.W. Hall, e-print quant-ph/0103072; e-print quant-ph/0107149.

[4] M.J.W. Hall and M. Reginatto, J. Phys. A 35, 3289 (2002); e-print quant-ph/0201084.

[5] N.C. Dias and J.N. Prata, Phys. Lett. A 291, 355 (2001).

[6] G.S. Agarwal and E. Wolf, Phys. Lett. A 26, 485 (1968); Phys.

Rev. Lett. 21, 180 (1968); G.S. Agarwal, Phys. Rev. 177, 400 (1969); K.E. Cahill and R.J. Glauber, ibid. 177, 1857 (1969);
177, 1882 (1969); G.S. Agarwal and E. Wolf, Phys. Rev. D 2 , 2161 (1970); 2, 2187 (1970); 2, 2206 (1970); J. Peřina, Coherence of Light (Reidel, Dordrecht, 1985); L. Mandel and E. Wolf, Optical Coherence and Quantum Optics (Cambridge University Press, Cambridge, 1995).

[7] L. Cohen, J. Math. Phys. 7, 781 (1966).

[8] N.C. Dias and J.N. Prata, Phys. Lett. A 302, 261 (2002).

[9] H. Margenau and R.N. Hill, Prog. Theor. Phys. 26, 722 (1961).

[10] C.H. Page, J. Appl. Phys. 23, 103 (1952); R. Gase, T. Gase, and K. Blüthner, Opt. Lett. 20, 2045 (1995).

[11] C.L. Mehta, J. Math. Phys. 5, 677 (1964).

[12] M. Hillery, R.F. O'Connell, M.O. Scully, and E.P. Wigner, Phys. Rep. 106, 121 (1984). 